\title{
Prevalencia de Cystoisospora suis en lechones lactantes y cerdas en Venezuela
}

\author{
Prevalence of Cystoisospora SUIS In Piglets and sows in Venezuela \\ Juan Carlos Pinilla León ${ }^{1,3}$, Natalia Da Silva Borges ${ }^{2}$
}

\section{Resumen}

Se determinó la prevalencia de Cystoisospora suis en lechones lactantes y cerdas madres en granjas porcinas de la región central de Venezuela. El estudio se condujo en granjas de tres tamaños y con antecedentes de diarrea neonatal: granjas pequeñas $(<150$ madres), medianas (150-500 madres) y grandes ( $>500$ madres). Se examinaron 572 camadas y sus madres. Las muestras se cultivaron en dicromato de potasio al $2.5 \%$ y procesadas mediante la técnica de flotación-centrifugación, utilizando una solución saturada de $\mathrm{NaCl}$ enriquecida con solución azucarada. C. suis se encontró en 55 granjas $(82.1 \%)$ y 210 camadas $(36.7 \%)$, con los mayores valores de prevalencia en las dos primeras semanas de vida $(\mathrm{p}<0.05)$, pero sin diferencias estadísticas entre tamaños de granja. El 9.4\% de las cerdas se encontraron infectadas y, de estas, el $89 \%$ de las camadas resultaron positivas a $C$. suis, dando una correlación significativa entre la positividad de lechones y sus madres $(\mathrm{r}=0.55 ; \mathrm{p}<0.05)$. Los resultados demuestran que $C$. suis se encuentra ampliamente distribuida en la región central de Venezuela, indistintamente del tamaño de la granja.

Palabras clave: cystoisosporosis; epidemiología; granjas; porcinos; Venezuela

\section{ABstRaCT}

The prevalence of Cystoisospora suis in lactating piglets and their dams in pig farms in the central region of Venezuela was determined. The study was conducted in farms of three sizes with a history of neonatal diarrhea: small ( $<150$ sows), medium (150-500 sows) and large ( $>500$ sows) farms. A total of 572 litters and their mothers were examined.

${ }^{1}$ Programa de Medicina Veterinaria, Facultad de Ciencias Agropecuarias, Universidad de Santander, Lagos de Cacique, Bucaramanga, Colombia

${ }^{2}$ Departamento de Parasitología Animal, Facultad de Agronomía, Universidad Rómulo Gallegos, Venezuela

${ }^{3}$ E-mail: j.pinilla@mail.udes.edu.com jcpinilla@hotmail.com

Recibido: 6 de julio de 2017

Aceptado para publicación: 8 de diciembre de 2017 


\begin{abstract}
Samples were cultured in $2.5 \%$ potassium dichromate and latter processed by the flotationcentrifugation technique using a $\mathrm{NaCl}$ saturaded solution. C. suis was found in 55 farms (82.1\%) and 210 litters (36.7\%), with the highest prevalence values in the first two weeks of life ( $<<0.05)$, but without statistical differences between farm sizes. Moreover, $9.4 \%$ of the sows were infected and of these, $89 \%$ of the litters were positive to $C$. suis, giving a significant correlation between the positivity of piglets and their mothers $(r=0.55 ; \mathrm{p}$ $<0.05$ ). The results show that $C$. suis is widely distributed in the central region of Venezuela, regardless of farm size.
\end{abstract}

Key words: cystoisosporosis; epidemiology; farms; swine; Venezuela

\section{INTRODUCCIÓN}

La cystoisosporosis porcina es una enfermedad intestinal causada por Cystoisospora suis (= Isospora suis), parásito Apicomplexa, con distribución cosmopolita y que se encuentra con mayor frecuencia en lechones lactantes a partir de la segunda semana de edad (Lindsay et al., 1999), y que puede ser controlada al mejorar las condiciones sanitarias de la granja (Lindsay y Dubey, 2005; Karamon et al., 2007).

Los animales desarrollan una diarrea amarillenta, inicialmente pastosa para hacerse fluida a $\operatorname{los} 2$ a 3 días (Lindsay et al., 1999). Karamon et al. (2007) señalaron $28 \%$ de prevalencia en camadas y $67 \%$ en granjas polacas, mientras que Niestrath et al. (2002) y Hamadejova y Vitovec (2005) encontraron 43 y $22 \%$ de prevalencia en camadas de Alemania y de la República Checa, respectivamente. Estudios realizados en Venezuela en granjas del municipio Mariño del estado Aragua, mostraron prevalencias de $19.8 \%$ en camadas y $60 \%$ en granjas (Da Silva y Pinilla, 2007), mientras que en el estado Carabobo se determinó $75 \%$ en granjas (González et al., 2000). Asimismo, Pinilla y Coronado (2008) señalaron $93.3 \%$ de prevalencia en granjas ubicadas en la región centro-occidental de Venezuela, y $31.6 \%$ en lechones lactantes y, posteriormente, Pinilla (2009) señaló $75 \%$ de prevalencia en granjas y $32 \%$ en camadas del estado Aragua.
Con respecto a la edad de los lechones, Sayd y Kawazoe (1996) y Hamadejova y Vitovec (2005) señalaron mayores valores de prevalencia en camadas de dos semanas, mientras que Niestrath et al. (2002) las encontraron en camadas de tres y cuatro semanas. Con respecto a la presencia del protozoario en cerdas madres, se reportan prevalencias de 2.3\% (Meyer et al., 1999), $13.2 \%$ (Pinilla y Coronado, 2008), 13.6\% (González, 1993) y 15\% (Karamon et al., 2007); sin embargo, varios trabajos de investigación no llegaron a encontrar excreción de ooquistes en cerdas madres (Lindsay et al., 1984, 1999; Stuart y Lindsay, 1988; Farkas et al., 2004).

El estrés del parto, sobre todo en cerdas primíparas, puede afectar la respuesta inmune de los animales (Klopfenstein et al., 1999; Lanfranchi et al., 2008). Por su parte, Shrestha et al. (2015) señalaron la posibilidad que las cerdas escondan formas evolutivas intra- y extra-intestinales del enterococcidio durante su crecimiento y que al primer parto pueden excretar los ooquistes, pero que conforme avance la edad, sus sistemas inmunológicos maduran contrarrestando la infección, al punto que la cantidad de ooquistes no sean detectables por técnicas parasitológicas convencionales.

Holm (2001) indica que el tamaño de la granja puede afectar la presencia del protozoario; sin embargo, se dispone de información contradictoria. Así, Farkas et al. (2004) 
y Estrada et al. (2004) señalaron que no existe asociación estadística entre $C$. suis con respecto al tipo y tamaño de la explotación, mientras que Niestrath et al. (2002) y Heylen et al. (2004) encontraron mayor presencia en granjas pequeñas y medianas, y Karamon et al. (2007) encontraron mayores valores en granjas grandes. Según Feporcina (2010), en la región central de Venezuela se encuentra más del 70\% del total de granjas intensivas del país y cerca de la mitad de vientres en producción. Por ello, el presente trabajo tuvo como objetivo determinar la prevalencia de Cystoisospora suis en lechones lactantes y cerdas en dicha región.

\section{Materiales y Métodos}

\section{Ubicación Geográfica}

El estudio se realizó en la región central de Venezuela. La zona presenta registros pluviométricos anuales entre 500 y $1450 \mathrm{~mm}$ y temperaturas medias anuales entre $24.5 \mathrm{y}$ $27{ }^{\circ} \mathrm{C}$ con $70 \%$ de humedad relativa. La región está situada entre 600 y $2400 \mathrm{msnm}$ (MARNR, 2007).

\section{Tipo y Manejo de las Granjas}

Se incluyeron explotaciones porcinas organizadas de ciclo completo. El manejo de las granjas es bueno; sin embargo, en su mayoría existen pobres condiciones higiénicosanitarias y antecedentes de diarrea neonatal. El tipo de animal pertenece a mestizos de líneas mejoradoras. La alimentación es con base a raciones balanceadas formuladas en plantas de alimentos próximas a las unidades de producción.

\section{Tamaño y Toma de Muestras}

Durante 2016 se examinaron 67 granjas porcinas con problemas de diarrea en el área de maternidad, que representan el 70\% del total de explotaciones ubicadas en la región central de Venezuela, según datos aportados por INSAI (2010). El tamaño de la muestra poblacional fue calculado mediante la fórmula descrita por Morales y Pino (1987), con una prevalencia conocida de $31.9 \%$ (Pinilla, 2009) y un nivel de confianza de 95\%. Se seleccionaron en forma aleatoria 572 camadas de diferentes edades juntamente con sus madres.

Las camadas se distribuyeron de manera proporcional en cada granja, según la cantidad de animales presentes. Las granjas fueron categorizadas como pequeñas $(n=17$; $<150$ madres), medianas ( $n=32 ; 150-500$ madres) y grandes ( $\mathrm{n}=18 ;>500$ madres). Se trabajó con 120 camadas de granjas pequeñas, 280 camadas de granjas medianas y 172 camadas de granjas grandes, además de las respectivas madres. Muestras fecales fueron colectadas de 4-5 lechones por camada haciendo un pool con las muestras. Para esto, se introdujeron hisopos por vía rectal para estimular la defecación. A las cerdas madres se les tomó la muestra fecal directamente de la ampolla rectal o del piso en caso hayan defecado durante el muestreo. Las muestras se llevaron en cavas refrigeradas a la Unidad de Investigación en Parasitología de la Facultad de Agronomía de la Universidad «Rómulo Gallegos», donde fueron conservadas en refrigeración hasta su procesamiento.

\section{Análisis Copro-Parasitológico}

Todas las muestras se cultivaron a temperatura ambiente en cápsulas de Petri utilizando $20 \mathrm{ml}$ de una solución de dicromato de potasio al 2.5\% durante $24 \mathrm{~h}$ (Hendrix, 1999). Luego, se utilizó la técnica de centrifugaciónflotación empleando una solución de azúcar y sal (1000 ml de solución saturada de $\mathrm{NaCl}$ + 500 g de azúcar, gravedad específica de 1.28) (Henriksen y Christensen, 1992) para realizar el diagnóstico parasitológico. En aquellas muestras donde la cantidad de grasa dificultaba la observación de ooquistes de $C$. suis, se empleó una técnica de sedimentación con PBS-éter descrita por Ortega-Mora et al. (1992). La visualización e identifica- 
ción de ooquistes se hizo con un microscopio binocular, usando magnificación de 10 y $40 \mathrm{X}$.

\section{Análisis Estadístico}

Los resultados se analizaron mediante la prueba de Chi cuadrado para determinar asociaciones estadísticas y la prueba de Fisher para establecer comparaciones. Para los cálculos se utilizó el programa estadístico Statistix.

\section{Resultados}

La prevalencia encontrada a $C$. suis fue de $82.1 \%$, donde el $36.7 \%$ de las camadas y el 9.4\% de las madres se encontraban infectadas (Cuadro 1), sin que hubiese diferencias significativas por efecto del tamaño de la granja.

En el Cuadro 2 se muestra una tabla de contingencia entre valores positivos y negativos de lechones lactantes y cerdas. De un total de 54 (9.4\%) cerdas infectadas a C. suis, se encontraron 48 (89\%) camadas positivas. La prueba de correlación de Pearson indicó una correlación significativa entre la positividad de lechones y sus madres $(r=0.55$; $\mathrm{p}<0.05$ ), lo que indica que al aumentar las madres positivas, aumenta la infección en los lechones.

En el Cuadro 3 se muestra la comparación entre los valores de prevalencia en las camadas de los cuatro grupos etarios en estudio, donde las camadas con 1 a 7 y 8 a 14 días mostraron los mayores valores de prevalencia $(\mathrm{p}<0.05)$ con respecto de aquellas con edades mayores (15 a 21 y >21 días).

\section{Discusión}

El $82.1 \%$ de prevalencia obtenido en el presente estudio fue relativamente similar a otros reportes en el país. Así, se reporta $75.5 \%$ en granjas de la región central (González et al., 2000), 75\% en granjas del estado Aragua (Pinilla, 2009) y 93.3\% en granjas ubicadas en la región centro-occidental del país (Pinilla y Coronado, 2008). Asimismo, fue similar al $82 \%$ reportado en granjas del sureste del estado de Sao Paulo, Brasil (Sayd y Kawazoe, 1996).

Es posible que esta parasitosis pudiera ser controlada de mejorarse las condiciones higiénico-sanitarias de los corrales de parición (Lindsay y Dubey, 2005; Karamon et al., 2007). Es de esperarse que el microclima existente en las unidades de parición proporcione las condiciones adecuadas de temperatura y humedad para el desarrollo de ooquistes de C. suis, en virtud de la resistencia de estos a los agentes físicos y químicos empleados en la preparación de los paritorios (Lindsay et al., 1999; Lindsay y Dubey, 2005; Karamon et al., 2007).

La tasa de prevalencia de ooquistes de C. suis en heces de lechones fue, asimismo, similar a reportes venezolanos (DaSilva y Pinilla, 2007; Pinilla y Coronado, 2008; Pinilla, 2009), aunque superiores al $21.8 \%$ reportado por González et al. (2000). No obstante, en este último caso, el empleo de la técnica de Wisconsin en el diagnóstico de C. suis pudo haber influido en sus resultados.

De la misma manera, estos resultados coinciden con los estudios de Sayd y Kawazoe (1996) y Hamadejova y Vitovec (2005), quienes señalaron mayores valores de prevalencia en camadas de dos semanas de edad, ya que la contaminación del paritorio provoca una elevada presión de infección, lo que trae como consecuencia que los animales se infecten en sus primeros días de vida. Si bien se puede observar una relativa variabilidad entre los valores de prevalencia reportados en el país, las técnicas copro-parasitológicas empleadas en este estudio fueron suficientes para estimar el comportamiento del parásito en camadas y, de esta manera, establecer programas de control en granjas (Pinilla, 2009). Kuhnert et al. (2006) demostró que la sensibilidad de la técnica de flotación con solución de azúcar-sal (53.3\%) fue más baja 
Cuadro 1. Prevalencia de Cystoisospora suis ${ }^{1}$ en lechones lactantes y cerdas madres en granjas porcinas de tres tamaños en Venezuela

\begin{tabular}{lccccc}
\hline $\begin{array}{l}\text { Tamaño de } \\
\text { granja }^{2}\end{array}$ & $\begin{array}{c}\text { Granjas } \\
\left(\mathrm{N} .{ }^{\circ}\right)\end{array}$ & $\begin{array}{c}\text { Granjas con } \\
\text { C. suis } \\
(\%)\end{array}$ & $\begin{array}{c}\text { Camadas } \\
\left(\mathrm{N} .^{\circ}\right)\end{array}$ & $\begin{array}{c}\text { Camadas } \\
\text { infectadas } \\
(\%)\end{array}$ & $\begin{array}{c}\text { Cerdas } \\
\text { infectadas } \\
(\%)\end{array}$ \\
\hline Pequeña & 17 & 82.4 & 120 & 42.5 & 12.5 \\
Mediana & 32 & 84.4 & 280 & 37.1 & 7.1 \\
Grande & 18 & 77.8 & 172 & 32 & 11 \\
\hline Total & 67 & 82.1 & 572 & 36.7 & 9.4 \\
\hline
\end{tabular}

${ }^{1}$ Sin diferencia significativa entre tamaños de granja

2 Pequeña: <150 madres; Mediana: 150-500 madres; Grande: >500 madres

Cuadro 2. Tabla de contingencia de positividad/negatividad a $C$. suis entre lechones lactantes y cerdas madres

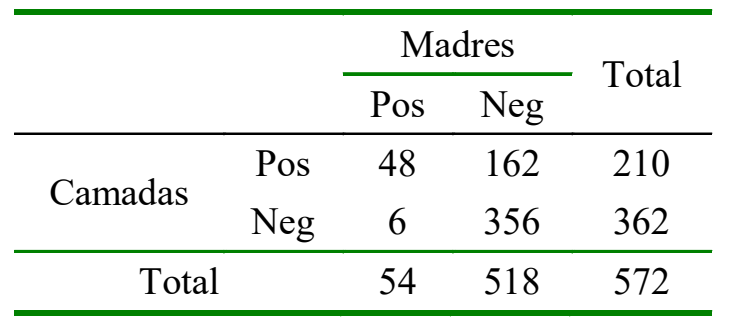

que la obtenida por microscopía de fluorescencia (83.3\%); sin embargo, para realizar esta técnica se requiere el microscopio de fluorescencia, contrario a la técnica de flotación. Por otro lado, diversos autores señalan que la sensibilidad de la técnica de flotación depende en gran medida del momento de la toma de la muestra (Niestrath et al., 2002; Lindsay et al., 2005; Pinilla y Coronado, 2008).

La excreción de ooquistes de $C$. suis en las madres lactantes fue observada en el $9.4 \%$ de los casos, encontrándose una correlación significativa entre madres y crías positivas al parásito. Se dispone de diversos estudios que demuestran la presencia de $C$. suis en cerdas lactantes (González, 1993; Meyer et al., 1999; DaSilva y Pinilla, 2007; Karamon et al., 2007; Pinilla, 2009), contrario a reportes donde no encontraron cerdas madres excretando ooquistes del enterococcidio (Stuart y Lindsay, 1988; Lindsay et al., 1999; Farkas et al., 2004). La baja presencia de ooquistes de $C$. suis en heces de madres demuestra que estos animales pueden excretar pequeñas cantidades de ooquistes no detectables con pruebas convencionales, que pueden tornarse infectivas y diseminarse por toda la paridera (Meyer et al., 1999). De esta forma, las cerdas podrían tener un rol importante en la cadena de transmisión, bien sea por un parasitismo activo no determinado, o por transporte pasivo de ooquistes a sus lechones.

Cuadro 3. Prevalencia (\%) de Cystoisospora suis en camadas de cuatro grupos etarios

\begin{tabular}{cccc}
\hline $\begin{array}{c}\text { Edad } \\
(\text { días })\end{array}$ & $\begin{array}{c}\text { Total } \\
(\mathrm{n})\end{array}$ & $\begin{array}{c}\text { Positivas } \\
(\mathrm{n})\end{array}$ & $\begin{array}{c}\text { Prevalencia } \\
(\%)\end{array}$ \\
\hline 1 a 7 & 138 & 55 & $39.9^{\mathrm{a}}$ \\
8 a 14 & 249 & 107 & $43.0^{\mathrm{a}}$ \\
15 a 21 & 138 & 32 & $23.2^{\mathrm{b}}$ \\
$>21$ & 47 & 16 & $34.0^{\mathrm{b}}$ \\
\hline Total & 572 & 210 & 36.7 \\
\hline a,b Superíndices diferentes indican & diferencia \\
significativa ( $\mathrm{p}<0.05)$ &
\end{tabular}


Probablemente, las cerdas durante su crecimiento mantienen y esconden formas evolutivas intra- y extra-intestinales del parásito, y cuando llega el momento del primer parto comienzan a excretar cantidades suficientes de ooquistes que son detectados por medio de técnicas de concentración-flotación; sin embargo, en la medida que avanzan en edad, sus sistemas inmunológicos se desarrollan hasta el punto de contrarrestar la infección y por tal motivo disminuye la cantidad de ooquistes, los cuales no son detectables por técnicas parasitológicas convencionales de flotación (Linsdsay et al., 1999; Lindsay y Dubey, 2005). También se podría pensar que cerdas primerizas se tornan más vulnerables que cerdas viejas a los cambios ocurridos en el traslado a la sala de partos, ruidos, personal y eventos del primer parto, ocasionando un gran estrés a estos animales que pudieran alterar su sistema inmunológico y de esta manera iniciar la excreción de ooquistes del parásito. Klopfenstein et al. (1999); Lanfranchi et al. (2008) señalaron que los nuevos eventos y cambios que ocurren en el parto de las cerdas (dolor, nerviosismo) son fuente principal de estrés, sobre todo en cerdas primíparas, las cuales también se pueden ver inmunocomprometidas por efecto de la lactancia.

La existencia de etapas de desarrollo extraintestinal de $C$. suis y otras vías de infección siguen siendo cuestionables y sin respuesta (Shrestha et al., 2015). La morfología de los ooquistes de C. suis, particularmente la falta de cuerpos de Stieda en los esporoquistes, sugiere la aparición de tales etapas en el ciclo de vida del protozoario (Karamon et al., 2007). Similar morfología de ooquistes es característica para el grupo de Cystoisospora que afectan perros y gatos, los cuales producen etapas extraintestinales (Karamon et al., 2007).

El tamaño de las granjas no afectó la presencia del protozoario. Los resultados obtenidos coinciden con lo señalado por Farkas et al. (2004) y Estrada et al. (2004), quienes no encontraron diferencias estadísticas entre la presencia de ooquistes de $C$. suis con respecto al tamaño de la explotación. Por el contrario, existen evidencias que señalan mayor presencia en granjas pequeñas (Heylen et al., 2004), aunque Niestrath et al. (2002) encontraron mayor prevalencia en granjas medianas, y Karamon et al. (2007) señalaron mayor prevalencia en granjas grandes. Probablemente, las pobres condiciones higiénicassanitarias de la mayoría de granjas examinadas tengan mayor efecto sobre la presencia del parásito.

\section{Agradecimientos}

Los autores agradecen al Programa de Medicina Veterinaria de la Facultad de Ciencias Agropecuarias de la Universidad de Santander por su valiosa colaboración y apoyo en la ejecución de este trabajo. Igualmente agradecen la colaboración prestada por el personal del Laboratorio de Investigación en Parasitología Veterinaria de la Universidad Rómulo Gallegos, Venezuela.

\section{Conclusiones}

- Existe una amplia distribución y prevalencia de Cystoisospora suis en la región central de Venezuela.

- Las cerdas madres podrían tener un rol de importancia en la cadena de transmisión de $C$. suis, pues su presencia en las heces puede afectar directamente a los lechones en las unidades de parición.

- El tamaño de la granja no tuvo efecto significativo sobre la prevalencia de $C$. suis.

\section{Literatura Citada}

1. Da Silva N, Pinilla J. 2007. Evaluación parasitológica y caracterización de granjas porcinas ubicadas en el municipio Santiago Mariño del estado Aragua. San Juan de Los Morros, Venezuela: Universidad Rómulo Gallegos. 54 p. 
2. Estrada E, Morilla A, Lafranchi E. 2004. Frequency of Isospora suis infected herds in Mexico. In: Proc $18^{\text {th }}$ IPVS Congress. Hamburg, Germany.

3. Farkas R, Szeidemann Z, Majoros G. 2004. Prevalence and geographical distribution of isosporosis in swine farms of Hungary. In: Proc $18^{\text {th }}$ IPVS Congress. Hamburg, Germany.

4. Feporcina. 2010. Comportamiento del sector porcino venezolano en el año 2005. Rev Inf Div 1: 10-12.

5. González Y. 1993. Prevalencia de coccidias en suinos del estado Aragua y Municipio Diego Ibarra del estado Carabobo. Vet Trop 18: 45-57.

6. González Y, Moreno L, García G. 2000. Isospora suis en granjas con diferentes condiciones de instalaciones y manejo. Vet Trop 25: 257-265.

7. Hamadejova K, Vitovec J. 2005. Ocurrence of the coccidium Isospora suis in piglets. Vet Med Czech 50: 159163.

8. Hendrix CM. 1999. Diagnóstico parasitológico veterinario. $2^{\mathrm{a}}$ ed. Madrid, España: Ed Harcourt Brace. 325 p.

9. Henriksen SA, Christensen JP. 1992. Demonstration of Isospora suis oocysts in faecal samples. Vet Rec 131: 443-444.

10. Heylen P, De Smet J, Vyt P, Gevaert D. 2004. Routine coprological confirmation of the history of Isosporosis in 10-18 day old piglets. Vlaams Diergeneesk Tijdschr 73: 420-423.

11. Holm A. 2001. Coccidiosis in piglets seen from the point of view of the practising veterinarian. Parasitol Res 87 : 357-359.

12. [INSAI] Instituto Nacional de Salud Agrícola Integral. 2010. Censo de granjas porcinas de los estados Aragua y Carabobo. En: Reportes de programa vacunación contra fiebre aftosa. Departamento de epidemiología.

13. Karamon J, Ziomko I, Cencek T. 2007. Prevalence of Isospora suis and Eimeria spp in suckling piglets and sows in Poland. Vet Parasitol 147: 171-175.
14. Klopfenstein C, Farmer C, Martineau G. 1999. Diseases of the mammary glands and lactation problems. In: Straw BE, D'Allaire S, Mengeling WL, Taylor DJ (eds). Diseases of swine. $8^{\text {th }}$ ed. Ames, Iowa, USA: Iowa State University Press. p 833-860.

15. Kuhnert $Y$, Schmäschke $R$, Daugschies A. 2006. Comparison of different methods for examining the feces of suckling piglets for Isospora suis. Berl Munch Tierarztl Wochenschr 119: 282-286.

16. Lanfranchi E, Gonxález J, Filios S. 2008. The efficacy of butafosfan in the periparturient sow - Field trial Mexico. In: Proc $20^{\text {th }}$ IPVS Congress. Durban, South Africa.

17. Lindsay D, Ernst J, Current W, Stuart B, Stewart T. 1984. Prevalence of oocysts of Isospora suis and Eimeria spp from sows on farms with and without a history of neonatal coccidiosis. J Am Vet Med Ass 185: 419-421.

18. Lindsay D, Blagburn B, Dubey J. 1999. Coccidia and other protozoa. Straw BE, D'Allaire S, Mengeling WL, Taylor DJ (eds). Diseases of swine. $8^{\text {th }}$ ed. Ames, Iowa, USA: Iowa State University Press. p 655-660.

19. Lindsay D, Dubey J. 2005. Coccidia and other protozoa. In: Straw BE, D'Allaire S, Mengeling WL, Taylor DJ (eds). Diseases of swine. $9^{\text {th }}$ ed. Ames, Iowa, USA: Iowa State University Press. p 861-873.

20. Meyer C, Joachim A, Daugschies A. 1999. Ocurrence of Isospora suis in larger piglet production units and on specialized piglet rearing farms. Vet Parasitol 82: 277-284.

21. [MARNR] Ministerio del Ambiente y de los Recursos Naturales Renovables. 2007. Anuario climatológico. Estados Aragua y Carabobo. Venezuela. $170 \mathrm{p}$.

22. Morales G, Pino A. 1987. Parasitología cuantitativa. Caracas: Acta Científica Venezolana. $132 \mathrm{p}$. 
23. Niestrath M, Takla M, Joachim A, Daugschies A. 2002. The role of Isospora suis as a pathogen in conventional piglet production in Germany. J Vet Med B Infect Dis Vet Public Health 49: 176-180.

24. Ortega-Mora L, Troncoso J, RojoVázquez F, Gómez-Bautista M. 1992. Evaluation of an improved method to purify Cryptosporidium parvum oocysts. Res Rev Parasitol 52: 127-130.

25. Pinilla JC. 2009. Prevalencia de Isospora suis en granjas porcinas intensivas ubicadas en el estado Aragua. Venezuela. Zootecnia Trop 27: 205-213.

26. Pinilla JC, Coronado A. 2008. Prevalencia de Isospora suis en lechones cria dos en granjas de la región Centro-Occidental de Venezuela. Zootecnia Trop 26: 47-53.

27. Sayd S, Kawazoe U. 1996. Experimental infection of swine by Isospora suis Biester 1934 for species confirmation. Mem Inst Oswaldo Cruz 93: 851-854. doi: 10.1590/S0074-02761998000600028

28. Shrestha A, Abd-Elfattah A, Freudens-chuss $B$, Hinney $B$, Palmieri N, Ruttkowski B, Joachim A. 2015. Cystoisospora suis - a model of mammalian cystoisosporosis. Front Vet Sci 2: 68. doi: 10.3389/fvets.2015.00068

29. Stuart B, Lindsay D. 1988. Coccidiosis in swine. Vet Clin Nor Am Food Anim Pract 2: 455-468. 